\title{
Enlarging the Domain of Convergence for Multiple Shooting by the Homotopy Method
}

\author{
Peter Lory \\ Institut für Mathematik der Technischen Universität München, Arcisstr. 21, D-8000 München 2, \\ Germany (Fed. Rep.)
}

\begin{abstract}
Summary. The homotopy method is a frequently used technique in overcoming the local convergence nature of multiple shooting. In this paper sufficient conditions are given that guarantee the homotopy process to be feasible. The results are applicable to a class of two-point boundary value problems. Finally, the numerical solution of two practical problems arising in physiology is described.
\end{abstract}

Subject Classifications: AMS(MOS): 65L10; CR: 5.17.

\section{Introduction}

Multiple shooting is a well-known method for the numerical solution of nonlinear two-point boundary value problems. It has been thoroughly tested in numerous realistic applications (see e.g. Bulirsch [2], Stoer and Bulirsch [25], Diekhoff et al. [8], and Keller [14]). Due to the nonlinearity of the problem, starting values sufficiently close to the true solution have to be available in order to start the process. A characteristic local convergence theorem is given in Weiss [28].

The present paper deals with a frequently used way in overcoming the local convergence nature of the iterative process, namely with the homotopy method (or continuation method). This technique takes advantage of the fact that most of the boundary value problems arising in applications depend on a physical parameter in a natural way. In general, this parameter $\tau$ may appear in the system of $n$ ordinary differential equations

$$
y^{\prime}=f(\tau, x, y) ; \quad x \in[a, b], \quad \tau \in[0,1]
$$

and in the two-point boundary conditions

$$
r(\tau, y(a), y(b))=0 .
$$

Here (1.1) is chosen so that for $\tau=1(1.1)$ is equivalent to the original problem. Mostly, (1.1) reduces to a somehow "familiar" problem for a certain value of the 
parameter, say $\tau=0$. Then a solution $y^{(0)}(x)$ of $(1.1)$ for $\tau=0$ is available. Starting with $\tau_{0}=0$, a homotopy chain of subproblems is solved taking the computed solution for $\tau=\tau_{i-1}$ as an initial approximation for the $i^{\text {th }}$ subproblem. If $\tau_{N}=1$ is reached (within a tolerable number $N$ of homotopy steps), the original problem is solved.

The general idea of using the homotopy method in actual computations of nonlinear equations seems to date back to the work of Lahaye [16]. Some authors (e.g. Roberts and Shipman [22], Wacker [26]) present theoretical estimates of constant stepsizes $\Delta \tau=\tau_{i}-\tau_{i-1}$, which guarantee the homotopy process to be successful. However, these estimates require the knowledge of computationally unavailable quantities and are by far too pessimistic in those parts of the $\tau$-interval where the solution depends on $\tau$ in an undramatic manner.

Having an efficient stepsize controlling algorithm available it is sufficient to prove the pure existence of a partition $0=\tau_{0}<\tau_{1}<\ldots<\tau_{N}=1$ so that the computed solution of the $(i-1)^{\text {th }}$ subproblem is contained in the domain of attraction for the next one. For nonlinear equations in $\mathbb{R}^{n}$ this has been carried out by Ortega and Rheinboldt [20], Avila [1], Menzel and Schwetlick [19]. The present paper treats the homotopy method for the problem (1.1) in connection with multiple shooting and gives sufficient conditions for its feasibility in terms of the original boundary value problem.

After some preliminaries in Sect.2, the feasibility of the homotopy method is studied in Sect.3. The results obtained there may be applied to a class of twopoint boundary value problems (Sect.4). This class contains as a special case a boundary value problem, which is used in physiology to model salt and water transport in epithelia. It is closely related to an extensive model of the renal counterflow system. Both problems were solved numerically, as described in Sect. 5. In these computations the stepsize control due to Deuflhard [6] was used successfully.

\section{Preliminaries}

The multiple shooting algorithm for the numerical solution of a two-point boundary value problem (1.1) is described in [25, 14] and realized in the code in Bulirsch, Stoer, and Deuflhard [4]. Here the interval $[a, b]$ is suitably subdivided

$$
a=x_{1}<x_{2}<\ldots<x_{m-1}<x_{m}=b \quad(m \text { nodes }) .
$$

Let $y\left(\tau, x ; x_{k}, s_{k}\right)(k=1, \ldots, m-1)$ denote the solution of the initial value problem

$$
y^{\prime}=f(\tau, x, y), \quad y\left(x_{k}\right)=s_{k}, \quad x \in\left[x_{k}, x_{k+1}\right] .
$$

The $n$-vectors $s_{k}$ have to be determined so that the following $n(m-1)$ conditions are satisfied:

continuity conditions (for $m>2$ ): 


$$
F_{k}\left(\tau, s_{k}, s_{k+1}\right):=y\left(\tau, x_{k+1} ; x_{k}, s_{k}\right)-s_{k+1}=0, k=1, \ldots, m-2 ;
$$

boundary conditions:

$$
F_{m-1}\left(\tau, s_{1}, s_{m-1}\right):=r\left(\tau, s_{1}, y\left(\tau, x_{m} ; x_{m-1}, s_{m-1}\right)\right)=0 .
$$

These conditions define a system of $n(m-1)$ nonlinear equations

$$
F(\tau, s):=\left(\begin{array}{c}
F_{1}\left(\tau, s_{1}, s_{2}\right) \\
\vdots \\
F_{m-2}\left(\tau, s_{m-2}, s_{m-1}\right) \\
F_{m-1}\left(\tau, s_{1}, s_{m-1}\right)
\end{array}\right)=0 \quad \text { with } s=\left(\begin{array}{c}
s_{1} \\
\vdots \\
s_{m-1}
\end{array}\right) .
$$

If $y^{(0)}(x)$ is a solution of $(1.1)$ for $\tau=0$ and $s_{k}^{(0)}:=y^{(0)}\left(x_{k}\right)$, the $n(m-1)$-vector $s^{(0)}$ satisfies $F(0,)=$.0 . Starting with this initial value, the homotopy $F$ defines the following continuation process: Let the partition

$$
0=\tau_{0}<\tau_{1}<\ldots<\tau_{N}=1
$$

suitably subdivide the interval $[0,1]$. Then for each subproblem $F\left(\tau_{i},.\right)=0 M_{i}$ Newton-iterates are computed (according to the use of Newton's method in $[4$, 14], and [25]):

$$
\begin{gathered}
s^{i, j+1}=s^{i, j}-\left[D_{s} F\left(\tau_{i}, s^{i, j}\right)\right]^{-1} F\left(\tau_{i}, s^{i, j}\right) \\
j=0, \ldots, M_{i}-1, \quad i=1, \ldots, N-1 .
\end{gathered}
$$

The starting values for these iterations are given by

$$
s^{1,0}=s^{(0)}, \quad s^{i+1,0}=s^{i, M_{i}}
$$

("classical predictor")

or

$$
\begin{aligned}
s^{1,0} & =s^{(0)}-\tau_{1} \cdot\left[D_{s} F\left(0, s^{(0)}\right)\right]^{-1} D_{\tau} F\left(0, s^{(0)}\right) \\
s^{i+1,0} & =s^{i, M_{i}}-\left(\tau_{i+1}-\tau_{i}\right) \cdot\left[D_{s} F\left(\tau_{i}, s^{i, M_{i}}\right)\right]^{-1} D_{\tau} F\left(\tau_{i}, s^{i, M_{i}}\right)
\end{aligned}
$$

(“Euler predictor").

(2.4) and (2.5) yield an $s^{N, 0}$, which is used as starting value for the final iteration

$$
\begin{gathered}
s^{N, j+1}=s^{N, j}-\left[D_{s} F\left(1, s^{N, j}\right)\right]^{-1} F\left(1, s^{N, j}\right) \\
j=0,1,2, \ldots
\end{gathered}
$$

Obviously the following definition according to [20] and [1] is meaningful.

Definition. If a partition (2.3) and $N-1$ integers $M_{1}, \ldots, M_{N-1}$ exist so that the process (2.4), (2.5.a) ((2.5.b) respectively) is well-defined and so that (2.6) converges to a solution of $F(1,)=$.0 , then the homotopy method (2.3)-(2.6) is called feasible with the classical predictor (the Euler predictor respectively).

Remark. The Euler predictor (2.5.b) represents an Euler step for the integration of Davidenko's differential equation (Davidenko [5]). At a first glance, the simplest continuation procedure seems to be integrating this differential equa- 
tion from $\tau=0$ to $\tau=1$. However, this approach would require the frequent evaluation of an explicitly not available right-hand side (cf. [6] and Feilmeier [11]). This argument and a comparison of the computing times in Feilmeier and Wacker [12] show that this method is very uneconomical.

\section{The Feasibility of the Homotopy Method}

In this section sufficient conditions for the feasibility of the homotopy method are given. The proof requires the following

Lemma. Let $\sigma:[0,1] \rightarrow R^{n(m-1)}$ be a continuous function with $\sigma(0)=s^{(0)}$. Define the stripe

$$
S(\Theta, \sigma):=\left\{(\tau, s) \mid \tau \in[0,1], s \in R^{n(m-1)},\|s-\sigma(\tau)\|<\Theta\right\}
$$

with some norm $\|\cdot\|$ in $R^{n(m-1)}$. Assume that the homotopy $F(\tau, s)$.

$F: S(\Theta, \sigma) \rightarrow R^{n(m-1)}$ satisfies the following conditions:

$$
F(\tau, \sigma(\tau))=0 \quad \text { for } \tau \in[0,1] .
$$

The functional matrix $D_{s} F(\tau, s)$ exists on $S(\Theta, \sigma)$ and is continuous there. $D_{s} F(\tau, \sigma(\tau))$ is nonsingular for $\tau \in[0,1]$.

Then the homotopy method (2.3)-(2.6) is feasible with the classical predictor.

If additionally $D_{\tau} F(\tau, s)$ exists on $S(\Theta, \sigma)$ and is continuous there, then the homotopy method is feasible with the Euler predictor.

The proof slightly extends that of Theorem 10.4.2 in [20] and is omitted here. It may be found in detail in [17].

Let the functions $f:[0,1] \times[a, b] \times \bar{G} \rightarrow R^{n}$ and $r:[0,1] \times \bar{G} \times \bar{G} \rightarrow R^{n}$ of the boundary value problem (1.1) be continuous. Here $G$ may denote an open, convex and bounded subset of $R^{n}$ and $\bar{G}$ its closure. Then

$$
\begin{aligned}
g(x) & =y^{\prime}(x)-f(\tau, x, y(x)), \\
w & =r(\tau, y(a), y(b))
\end{aligned}
$$

defines a homotopy $H(\tau, y)$

$$
H:[0,1] \times \bar{D} \rightarrow C_{0}[a, b] \times R^{n}
$$

with $D:=\left\{y \in C_{1}[a, b] \mid y(x) \in G\right.$ for every $\left.x \in[a, b]\right\}$.

As usual $C_{1}[a, b]$ is equipped with the norm $\|y\|_{1}:=\max \left(\|y\|_{0},\left\|y^{\prime}\right\|_{0}\right)$, where $\|\cdot\|_{0}$ is the maximum norm.

Theorem. Suppose that in addition to the above assumptions the partial derivative $D_{y} f(\tau, x, y)$ exists on $[0,1] \times[a, b] \times G$ and is continuous there and that the same is valid for $D_{u} r(\tau, u, v)$ and $D_{v} r(\tau, u, v)$ on $[0,1] \times G \times G$. Assume that 
if $y_{0} \in D$ is a solution of $H\left(\tau_{0},.\right)=0$, then the linearized problem

$y^{\prime}(x)-D_{y} f\left(\tau_{0}, x, y_{0}(x)\right) \cdot y(x)=0$

$D_{u} r\left(\tau_{0}, y_{0}(a), y_{0}(b)\right) \cdot y(a)+D_{v} r\left(\tau_{0}, y_{0}(a), y_{0}(b)\right) \cdot y(b)=0$

has only the trivial solution $y \equiv 0$;

$H(\tau,)=$.0 has no solution on the boundary of $D$ for $\tau \in[0,1]$.

Then, if $y^{(0)} \in D$ is a solution of $H(0,)=$.0 , there exists one and only one continuous function $\eta:[0,1] \rightarrow D$ with $\eta(0)=y^{(0)}$ and $H(\tau, \eta(\tau))=0$ for $\tau \in[0,1]$. Additionally, the homotopy method (2.3)-(2.6) is feasible with the classical predictor and with $s^{(0)}=\left(y^{(0)}\left(x_{1}\right), \ldots, y^{(0)}\left(x_{m-1}\right)\right)^{T}$.

Sketch of the proof' (for details see [17]). The implicit function theorem and (3.5) guarantee that a solution $\tilde{\eta}(\tau)$ with $H(\tau, \tilde{\eta}(\tau))=0$ for $\tau \in[0, \alpha](0 \leqq \alpha<1)$ may be continued locally. The so-called continuation property (see Rheinboldt [21]) can be proved by the Arzelà-Ascoli theorem and (3.6). Then a standard technique along the lines of Theorem 2.4 in [21] demonstrates the first part of the statement.

In order to prove the second part, the above lemma is applied. To this purpose the continuous function $\sigma:[0,1] \rightarrow R^{n(m-1)}$ is defined by

$$
\sigma(\tau)=\left(\begin{array}{c}
\sigma_{1}(\tau) \\
\vdots \\
\sigma_{m-1}(\tau)
\end{array}\right):=\left(\begin{array}{c}
(\eta(\tau))\left(x_{1}\right) \\
\vdots \\
(\eta(\tau))\left(x_{m-1}\right)
\end{array}\right)
$$

Evidently $\sigma(0)=s^{(0)}$, and a detailed investigation shows that $F(\tau, s)$ (see (2.2)) is defined on a stripe $S(\Theta, \sigma)$. (3.1) is obvious and (3.2) follows from the fact, that the solution of an initial value problem depends on its initial values in a smooth manner (e.g. Walter [27]).

In order to demonstrate (3.3), let $\tau=\tau_{0}$ and $E:=A+B G_{m-1} \ldots G_{1}$ where

$$
\begin{aligned}
& G_{k}:=D_{s_{k}} y\left(\tau_{0}, x_{k+1} ; x_{k}, s_{k}\right) \quad \text { with } s_{k}:=\sigma_{k}\left(\tau_{0}\right), \quad k=1, \ldots, m-1 ; \\
& A:=D_{u} r\left(\tau_{0}, \sigma_{1}\left(\tau_{0}\right), \sigma_{m}\left(\tau_{0}\right)\right) \quad\left(\sigma_{m}(\tau):=(\eta(\tau))(b)\right) ; \\
& B:=D_{v} r\left(\tau_{0}, \sigma_{1}\left(\tau_{0}\right), \sigma_{m}\left(\tau_{0}\right)\right) .
\end{aligned}
$$

The chain rule yields

$$
E=A+B \cdot D_{s_{1}} y\left(\tau_{0}, b ; a, \sigma_{1}\left(\tau_{0}\right)\right)
$$

and this matrix is proved to be nonsingular in [28].

$\operatorname{As} \operatorname{det}(E)=\operatorname{det}\left(D_{s} F\left(\tau_{0}, \sigma\left(\tau_{0}\right)\right)\right)$ (see [25]), (3.3) is shown.

Corollary. Suppose that in addition to the hypotheses of the theorem $D_{\tau} f(\tau, x, y)$ exists on $[0,1] \times[a, b] \times G$ and is continuous there and that the same is valid for $D_{\tau} r(\tau, u, v)$ on $[0,1] \times G \times G$. Then the conclusions of the theorem are right for the Euler predictor, too.

Remark. For the sake of brevity the functions $f, D_{y} f$, and $D_{\tau} f$ were assumed to be continuous in $x$. A more detailed investigation shows that in the above theorem and in the corollary piecewise continuity would be sufficient (see [17]). 


\section{Application to a Class of Boundary Value Problems}

In order to illustrate the results of the preceding section, consider the following nonlinear boundary value problem, which is used in physiology to model salt and water transport in epithelia (see Sect. 5):

$$
\begin{aligned}
&-D \cdot C^{\prime \prime}(x)+(v(x) \cdot C(x))^{\prime}=f(x), \\
& v^{\prime}(x)+\tau \cdot J(x, C(x))=0, \quad 0<x<1, \\
& C^{\prime}(0)=0, \quad C(1)=a, \quad v(0)=0 .
\end{aligned}
$$

Here $D$ and a are positive constants and the parameter $\tau$ varies in $[0,1]$. The function $f(x)$ is assumed to be continuous and nonnegative. $J(x, C)$ is continuous and continuously differentiable with respect to the second variable and $J_{C}(x, C)<0$.

To apply the theorem, note that

$$
C_{0}(x)=a+D^{-1} \cdot \int_{x}^{1} f_{1}(t) d t, \quad v_{0}(x)=0, \quad\left(f_{1}(x):=\int_{0}^{x} f(t) d t\right)
$$

is the only solution of (4.1) for $\tau=0$.

(3.5) is trivial for $\tau_{0}=0$ and is demonstrated in Kellogg [15] for $\tau_{0}>0$. For the proof of (3.6), let $C_{\tau}(x), v_{\tau}(x)$ be a solution of (4.1), where the subscript indicates the dependence on the parameter $\tau$. Then it may be shown analogously to [15] that $0<C_{\tau}(x)<K$ with a constant $K$, which is independent of $\tau$. As a consequence,

$$
-\tau \cdot \int_{0}^{x} J(t, 0) d t<v_{\tau}(x)<-\tau \cdot \int_{0}^{x} J(t . K) d t
$$

and $\left|v_{\tau}(x)\right|<M_{3}$ with a suitably defined constant $M_{3}$. Finally, there exists a constant $M_{2}$ with $\left|C_{\tau}^{\prime}(x)\right|<M_{2}$. To see this, integrate the first differential equation in (4.1) to obtain

Defining

$$
C_{\tau}^{\prime}(x)=D^{-1} \cdot\left[C_{\tau}(x) \cdot v_{\tau}(x)-f_{1}(x)\right] .
$$

$$
G:=\left\{y \in R^{3}\left|0<y_{1}<K,\right| y_{2}\left|<M_{2},\right| y_{3} \mid<M_{3}\right\}
$$

(3.6) follows immediately.

Conclusion. Applying the homotopy method to the boundary value problem (4.1), the feasibility with both the classical and the Euler predictor is guaranteed.

\section{Numerical Examples Arising in Physiology}

The following two-point boundary value problems were solved by the program BSHOM. This algorithm is based on the multiple shooting code in [4] and is equipped with an automatic control of the homotopy stepsizes due to [6]. In 
addition to the solution it computes a special norm of the matrix $E$ (see Sect. 3). This norm $(E)$ represents the sensitivity of the problem relative to the variation of $s_{1}$. The integration of the initial value problems (2.1) was performed by the routines DIFSY1 (Bulirsch and Stoer [3], stepsize control: Hussels [13]) and RKF7 (Fehlberg [10]). All these programs were run on the computers of the Leibniz-Rechenzentrum der Bayerischen Akademie der Wissenschaften.

\section{Example 1. Water and Solute Transport in Epithelia}

Most epithelia absorb or secrete specific fluids, such as bile, gastric juice, sweat, and saliva. At the ultrastructural level, they possess long, narrow channels open at one end and closed at the other. According to Diamond and Bossert [7] the water and solute transport in such a channel may be described by the following boundary value problem:

$$
\begin{aligned}
& -D \cdot C^{\prime \prime}(x)+(v(x) \cdot C(x))^{\prime}=(2 / r) \cdot N(x), \\
& v^{\prime}(x)+(2 / r) \cdot P \cdot\left(C_{0}-C(x)\right)=0, \\
& C^{\prime}(0)=0, \quad C(L)=C_{0}, \quad v(0)=0 .
\end{aligned}
$$

The dependent variables $C(x)$ and $v(x)$ represent the concentration and the velocity of the fluid flow at height $x$ in a cylindrical channel of length $L$ and radius $r$. The concentration $C_{0}$ outside the channel, the water permeability $P$, and the diffusion coefficient $D$ are positive constants. The rate of active solute transport $N(x)$ is a nonnegative step function:

$$
N(x)=\left\{\begin{array}{cc}
N_{0} & \text { for } 0 \leqq x<L / 10 \\
0 & \text { for } L / 10 \leqq x \leqq L .
\end{array}\right.
$$

Substituting $\tau \cdot P$ for $P$ with $\tau \in[0,1]$ the water permeability is varied as a natural parameter. Considering the remark at the end of Sect. 3 (5.1) is contained in the class of boundary value problems that was investigated in Sect. 4 . Therefore, the homotopy method is feasible.

The computations were performed on a TR440 in single precision arithmetic. The physiological values for $N_{0}$ and $P$ cover the following ranges:

$$
1_{10^{-10}} \leqq N_{0} \leqq 1 \cdot 10^{-5}, \quad 1_{10^{-6}} \leqq P \leqq 2 \cdot 10^{-4} .
$$

In [7] solutions are computed for $P=2.10^{-5}$ with $N_{0}=1_{10^{-5}}, C_{0}=0.3, D$ $=1.10^{-5}, L=0.01, r=5.10^{-6}$. In the present paper $P$ is raised to $2 \cdot 10^{-4}$. As a result the sensitivity of the problem increases significantly as indicated in the table. 
Table 1. Computation of (5.1) by the homotopy method using the classical predictor

$\mathrm{NHS}=$ number of homotopy steps $\Delta \tau_{\max } / \Delta \tau_{\min }=$ ratio of the maximal to the minimal homotopy stepsize

\begin{tabular}{lcll}
\hline$P=$ & $N H S$ & $\Delta \tau_{\max } / \Delta \tau_{\min }$ & $\operatorname{norm}(E)$ \\
\hline $2 \cdot 10^{-5}$ & 5 & 11 & $8 \cdot 10^{4}$ \\
$5 \cdot 10^{-5}$ & 7 & 23 & $2 \cdot 10^{6}$ \\
$1 \cdot 10^{-4}$ & 7 & 64 & $8 \cdot 10^{7}$ \\
$2 \cdot 0^{-4}$ & 10 & 149 & $6 \cdot 10^{9}$ \\
\hline
\end{tabular}

Remark. Performing the shooting method in backward direction norm $(E)$ may be reduced to $2 \cdot 1_{0^{4}}$ (for $\left.P=2 \cdot 10^{-4}\right)$, whereas the computing time remains nearly unchanged.

\section{Example 2. Kidney Model}

According zu Stephenson, Tewarson, and Mejia [24] and Stephenson, Mejia, and Tewarson [23] the solute and water movement in the kidney may be described by an extensive boundary value problem with the following differential equations:

where

$$
\left.\begin{array}{l}
d F_{i v} / d x=-J_{i v}, \\
d F_{i k} / d x=-J_{i k}, \\
F_{i k}=F_{i v} \cdot c_{i k}-D_{i k} \cdot d c_{i k} / d x,
\end{array}\right\} \begin{gathered}
i=1, \ldots, 6 \\
k=1,2
\end{gathered}
$$

$$
\begin{aligned}
& J_{i v}=h_{i v} \cdot\left[\left(c_{61}-c_{i 1}\right)+\left(c_{62}-c_{i 2}\right)\right], \\
& J_{i k}=h_{i k} \cdot\left(c_{i k}-c_{6 k}\right)+a_{i k} /\left(1+b_{i k} / c_{i k}\right), \\
& J_{3 v}=h_{3 v} \cdot\left[\left(c_{01}-c_{31}\right)+\left(c_{02}-c_{32}\right)\right], \\
& J_{3 k}=h_{3 k} \cdot\left(c_{3 k}-c_{0 k}\right)+a_{3 k} /\left(1+b_{3 k} / c_{3 k}\right), \\
& J_{6 v}=J_{1 v}+J_{2 v}+J_{4 v}+J_{5 v}, \\
& J_{6 k}=J_{1 k}+J_{2 k}+J_{4 k}+J_{5 k} .
\end{aligned}
$$

This problem seems to be too complicated for a rigorous application of the theory of Sect. 3. However, (5.1) may be regarded as a strongly reduced version of (5.2). So it appears reasonable to attack the kidney model with the same homotopy that was used in Example 1. Therefore, the three water permeability coefficients $h_{1 v}, h_{3 v}$, and $h_{4 v}$ were chosen as natural homotopy parameters.

The numerical solution was performed on a CDC Cyber 175 (double precision) using the parameter set given in Farahzad and Tewarson [9]. Taking advantage of some trivial parameters the number of differential equations may be reduced to 13 (see [17] and [18]). The problem was solved in 31 (classical) homotopy steps from permeabilities $(0 ., 0 ., 0$.) up to $(10 ., 1 ., 10$.). In the course of the computations, norm $(E)$ increased from $3 ._{10^{7}}$ to $4_{10^{12}}$ indicating an extreme sensitivity of the problem. The homotopy stepsizes were spread by a factor of 
about 530. The prescribed relative accuracy was eps $={ }_{10^{-5}}$ for the intermediate subproblems and eps $={ }_{10^{-12}}$ for the final problem. As the algorithm performed an additional Newton step, after it had reached this accuracy, the continuity and boundary conditions were satisfied with a precision of ${ }_{10^{-31}}$ for the final results. An important check is the conservation of mass which was computed to hold to a relative accuracy of ${ }_{10^{-24}}$. Further details are to be found in [17] and [18].

Acknowledgement. The author wishes to thank R. Bulirsch who stimulated and encouraged this work.

\section{References}

1. Avila, J.H.: The feasibility of continuation methods for nonlinear equations. SIAM J. Numer. Anal. 11, 102-122 (1974)

2. Bulirsch, R.: Die Mehrzielmethode zur numerischen Lösung von nichtlinearen Randwertproblemen und Aufgaben der optimalen Steuerung. Report der Carl-Cranz-Gesellschaft, 1971

3. Bulirsch, R., Stoer, J.: Numerical treatment of ordinary differential equations by extrapolation methods. Numer. Math. 8, 1-13 (1966)

4. Bulirsch, R., Stoer, J., Deuflhard, P.: (1980) Numerical solution of nonlinear two-point boundary value problems I. Numer. Math., Handbook Series Approximation (in preparation)

5. Davidenko, D.: On a new method of numerical solution of systems of nonlinear equations. Dokl. Akad. Nauk SSSR 88, 601-602 (1953)

6. Deuflhard, P.: A stepsize control for continuation methods and its special application to multiple shooting techniques. Numer. Math. 33, 115-146 (1979)

7. Diamond, J.M., Bossert, W.H.: Standing-gradient osmotic flow. J. Gen. Physiol. 50, 2061-2083 (1967)

8. Diekhoff, H.-J., Lory, P., Oberle, H.J., Pesch, H.-J., Rentrop, P., Seydel, R.: Comparing routines for the numerical solution of initial value problems of ordinary differential equations in multiple shooting. Numer. Math. 27, 449-469 (1977)

9. Farahzad, P., Tewarson, R.P.: An efficient numerical method for solving the differential equation of renal counterflow systems. Comput. Biol. Med. 8, 57-64 (1978)

10. Fehlberg, E.: Klassische Runge-Kutta Formeln fünfter und siebenter Ordnung mit Schrittweitenkontrolle. Computing 4, 93-106 (1969)

11. Feilmeier, M.: Numerische Aspekte bei der Einbettung nichtlinearer Probleme. Computing 9 , 355-364 (1972)

12. Feilmeier, M., Wacker, H.J.: Zur numerischen Praxis von Einbettungsmethoden. Z. Angew. Math. Mech. 52, T 200-T 202 (1972)

13. Hussels, H.G.: Schrittweitensteuerung bei der Integration gewöhnlicher Differentialgleichungen mit Extrapolationsverfahren. Universität zu Köln, Mathematisches Institut, Diplomarbeit, 1973

14. Keller, H.B.: Numerical methods for two-point boundary value problems. London: Blaisdell 1968

15. Kellogg, R.B.: Uniqueness in the Schauder fixed point theorem. Proc. Amer. Math. Soc. 60, 207210 (1976)

16. Lahaye, E.: Une méthode de résolution d'une catégorie d'équations transcendantes. C.R. Acad. Sci. Paris 198, 1840-1842 (1934)

17. Lory, P.: Homotopieverfahren und Anwendung der Mehrzielmethode auf mathematische Modelle aus der Physiologie. Technische Universität München, Institut für Mathematik, Dissertation, 1978

18. Lory, P.: Numerical solution of a kidney model by multiple shooting. Math. Biosci. (in press, 1980)

19. Menzel, R., Schwetlick, H.: Zur Lösung parameterabhängiger nichtlinearer Gleichungen mit singulären Jacobi-Matrizen. Numer. Math. 30, 65-79 (1978) 
20. Ortega, J.M., Rheinboldt, W.C.: Iterative solution of nonlinear equations in several variables. New York: Academic Press 1970

21. Rheinboldt, W.C.: Local mapping relations and global implicit function theorems. Trans. Amer. Math. Soc. 138, 183-198 (1969)

22. Roberts, S.M., Shipman. J.S.: Two-point boundary value problems: Shooting methods. New York: Elsevier 1972

23. Stephenson, J.L., Mejia, R., Tewarson, R.P.: Model of solute and water movement in the kidney. Proc. Nat. Acad. Sci. USA 73, 252-256 (1976)

24. Stephenson, J.L., Tewarson, R.P., Mejia, R.: Quantitative analysis of mass and energy balance in non-ideal models of the renal counterflow system. Proc. Nat. Acad. Sci. USA 71, 1618-1622 (1974)

25. Stoer, J., Bulirsch, R.: Introduction to numerical analysis. Berlin-Heidelberg-New York: Springer 1980

26. Wacker, H.J.: Ein Iterationsverfahren zur Lösung spezieller nichtlinearer Randwertprobleme. Computing 9, 275-291 (1972)

27. Walter, W.: Gewöhnliche Differentialgleichungen. Heidelberger Taschenbuch, Band 110. BerlinHeidelberg-New York: Springer 1976

28. Weiss, R.: The convergence of shooting methods. BIT 13, 470-475 (1973)

Received December 21, 1979 